\title{
Gestión de seguridad y salud ocupacional y riesgos laborales en una empresa constructora del Perú
}

\section{Occupational health and safety management and occupational risks in a construction company in Peru}

DOI: $10.46932 / \mathrm{sfjdv} 2 \mathrm{n} 4-018$

Received in: March 1st, 2021

Accepted in: May 30th, 2021

\author{
Jossimar Ortega Aliaga \\ Filiación: Universidad César Vallejo \\ Correo: civil_1010@hotmail.com \\ Rubén Moisés Mauricio Avalos \\ Filiación: Universidad César Vallejo \\ Correo: mauriciodata@gmail.com \\ Jimmy Edison Macedo Bedoya \\ Filiación: Universidad César Vallejo \\ Correo: JIMMYEDY30@gmail.com \\ César Orlando Yumpo Chuquilin \\ Filiación: Universidad César Vallejo \\ Correo: coych13@hotmail.com
}

\section{RESUMEN}

La finalidad de la investigación fue determinar la relación entre la gestión de seguridad y salud ocupacional y los riesgos laborales en la empresa constructora Cobra Perú, San Isidro,2020. Su tipo básico, diseño correlacional, transversal. La población es de 82 colaboradores que laboran en la empresa. Se emplearon encuestas como instrumento de recolección de información la cual nos permitió conocer las actitudes y opiniones de todos los colaboradores. De tal manera se realizó la validación de contenido mediante la validez de juicios de expertos y de confiabilidad para la variable Gestión de la seguridad y salud ocupacional obteniendo un alfa de Cronbach de 0.947 y para la variable Riesgos Laborales de 0.941 implicando solidez interna aplicada, Se aplicaron cuestionarios validados y confiables. Los resultados fueron que la gestión de seguridad y salud ocupacional se relaciona con los riesgos laborales moderadamente con un (Rho 0,472 y p-valor 0,000 ).

Palabras clave: Gestión, seguridad, salud, riesgo, laboral.

\footnotetext{
ABSTRACT

The purpose of the investigation was to determine the relationship between occupational health and safety management and occupational risks at the construction company Cobra Peru, San Isidro, 2020. Its basic type, correlational, transversal design. The population is 82 collaborators who work in the company. A survey was used as an instrument to collect information which allows us to know the attitudes and opinions of all the collaborators (Valderrama, 2015). In this way, content validation was carried out through the validity of expert judgments and reliability for the variable. Occupational health and safety management,
} 


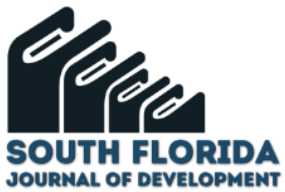

obtaining a Cronbach alpha of 0.947 and for the Occupational Risks variable of 0.941 , implying applied internal strength. Validated and reliable questionnaires were applied. The results were that occupational health and safety management is related to occupational risks moderately with a (Rho 0.472 and p-value 0.000).

Keywords: Management, security, health, risk, work.

\section{INTRODUCCIÓN}

Alrededor del mundo la gran mayoría de empresas pertenecientes a la rama de la construcción están como las causantes de accidentes, enfermedades e impactos negativos al medio ambiente, esto es debido al escaso cumplimiento, sumado a la escasa planificación en temas de gestión de seguridad y salud en el trabajo, este inconveniente también se pone evidente al carecer de colaboradores en buen estado de salud se ve evidenciado en el bajo desempeño en el trabajo y por ello la disminución en la productividad y por ende la rentabilidad de las empresas. La gran mayoría de causantes de los accidentes en el centro laboral son causadas por el entorno, las condiciones y carencia de procedimientos seguros, concepción inadecuada del trabajo, la inexistencia de capacitación que permita a los trabajadores emplear adecuadamente las herramientas y nuevas tecnologías en el centro de trabajo, a esto también se suma el cansancio y fatiga que en su gran mayoría son ocasionados por extensas horas en el centro de trabajo desempeñando la misma actividad, todo ello son factores que conllevan a la ocurrencia de accidentes.

En Perú, las empresas del sector industrial están especialmente atentas a mejorar los aspectos de seguridad. La gestión adecuada de la seguridad y salud ocupacional es un objetivo estratégico comercial clave de las empresas entrevistadas. En particular, la gestión de la construcción, especialmente los avances en seguridad ocupacional y las aplicaciones posteriores, son raras y poco prácticas. Esto se debe a que los empleados aún no tienen una cultura de prevención. Porque todavía están considerando el tema de la seguridad laboral como gasto; se debe considerar como inversión a largo plazo.

La constructora Cobra Perú ubicada en el distrito de San Isidro ofrece soluciones en temas de construcción, instalación y mantenimiento de instalaciones de alta tensión, equipamientos ferroviarios, sistemas de comunicación y servicios especiales para el mantenimiento integral de todo tipo de infraestructura y sus edificaciones. Además de los trabajos en altura, Cobra Perú también realiza trabajos preparatorios como la apertura de trincheras con riesgo de caída a diferentes alturas y riesgo de colapso, en particular para trabajos transportados. Por lo tanto, para salir durante la gestión de cables activos de alta tensión, los asociados de la empresa en cuestión verificarán los documentos reglamentarios y emprenderán acciones legales, así como tomarán e implementarán medidas preventivas, incluida la gestión e información en documentos relacionados. a varios eventos. Existen algunas restricciones para. 
Las enfermedades pueden ocurrir en el lugar de trabajo. Aunque implementado en el Sistema que permita la gestión adecuada de la seguridad y salud ocupacional, aún existen numerosas limitaciones, que se ponen en manifiesto en las distintas obras que se están construyendo.

Refiérase los siguientes autores como parte de la investigación internacional realizada. Roa (2017) en su trabajo de investigación titulado Gestión de Seguridad y Salud Ocupacional, un objetivo común definido es implementar avances en los sistemas de administración de seguridad y salud en el trabajo. Utilizó el método de análisis analítico para este propósito. Empleo muestras de 42 empresas constructoras. Las conclusiones obtenidas evidencian una correlación importante entre las dos variables. Cabe mencionar que no todos. De igual forma, el llamado SGSST afirma que en casi todos los casos no se encuentran preparados para hacer suficiente trabajo de planificación para lograr el desempeño y lograr el resultado final es una correlación positiva. Significancia estadística. En resumen, concluimos que las empresas son interdependientes y deben lograr e implementar interacciones de manera unificada en todas las etapas del ciclo PHVA.

Por todo esto, Nkrumah, Gyabeng y Akoto (2020) realizan un estudio sobre el avance de los marcos regulatorios en temas de salud y seguridad ocupacional relacionado a la industria del petróleo y el gas de Ghana, limitada al sector de administración de la salud y seguridad laboral. Sea consciente de las consecuencias. Se han establecido normas de la Directiva de seguridad para prevenir la posibilidad de accidentes en el lugar de trabajo en varias empresas, con un enfoque particular en las empresas de petróleo y gas. Empleados, colaboradores, como se concluye a partir de un estudio descriptivo transversal y encuesta a 699 empleados que estas dos variables no son muy positivas y no tienen una relación razonablemente significativa con respecto al riesgo potencial.

En relación con trabajos de investigación nacional, Fabian (2017) tuvo como meta disminuir los riesgos para la salud ocupacional de esta planta concentradora de Yauris, con la finalidad de mejorar la seguridad y salud ocupacional en la planta de Yauris. El estudio fue correlacional descriptivo cuantitativo, para conseguir tal propósito aplicó una encuesta a 10 personas que ayudaron a ajustar la efectividad de este sistema de seguridad. Al final, llego a la conclusión de que un sistema de gestión de la seguridad y salud ocupacional, puede mejorar la eficiencia laboral de los empleados.

Poyehuanca (2019) Realizó un estudio de los sistemas de administración de seguridad y salud ocupacional de Norpuno. Propone que un sistema de administración de salud ocupacional previene los riesgos en la salud y seguridad, reduce drásticamente el número de accidentes y mejora el desempeño laboral. Para lograrlo, desarrolló una investigación de tipo descriptiva de correlación, se realizó una encuesta mediante la aplicación de una herramienta denominada cuestionario para recopilar información. 
Finalmente, concluye que existe una clara relación entre las dos variables, por lo que resulta gravitante trabajar y desplegar el sistema.

\section{MARCO TEÓRICO}

En concordancia entre la variable gestión de seguridad y salud ocupacional, al lidiar con altibajos en temas de seguridad en el lugar de trabajo, como ensamblar, clasificar y usar equipos, es importante que la toma de decisiones cumpla con los estándares. Reciba criterios y resultados vinculantes. Su función principal es realizar evaluaciones con la finalidad de mejorar los resultados de prevención de accidentes en el centro laboral. Entonces, es una herramienta que le permite monitorear su progreso, encontrar áreas de mejora y verificar su progreso. Una de sus cualidades es la disposición de adaptarse a los futuros cambios. (ISEM, 2011) Para las características, puestos o medidas de seguridad establecidas, esto no está relacionado a un sistema de procedimientos de trabajo, sino también requiere que las empresas protejan a sus empleados y al medio ambiente para brindar el desempeño requerido. (Mears, Sean y Rhona, 2003).

Teniendo en cuenta lo que está haciendo, recuerde que el lugar de trabajo conlleva riesgos que no sólo son relevantes desde este punto de vista, sino que cambian con el tiempo y afectan la seguridad de los trabajadores (Arévalo y Molano, 2013). En relación con la dimensión primera que es implementación y operación, este aspecto es la relación que guarda la planificación de la formación, las acciones preventivas, la previsión de emergencias, el asesoramiento y la presentación de informes, y las responsabilidades de las organizaciones y de cada empleador en su implementación y funcionamiento, comité de condiciones de trabajo y sus métodos de gestión (Apaza, 2012).

En relación con la segunda dimensión, se deben conocer requisitos legales y verificables para asegurar la salud y seguridad de los empleados. De lo contrario, deben implementarse en la vida cotidiana (Merino, 2016). De acuerdo con la tercera dimensión, se tiene en cuenta la gestión de documentos e información, la investigación de accidentes y enfermedades profesionales, y se señalan los peligros o situaciones que amenazan la probabilidad de ocurrencia, indicando las responsabilidades que debe tener todo miembro de un grupo (Robson, 2010).

En tal sentido reviste vital importancia porque se puede definir de varias formas, por lo tanto, estas medidas se denominan medidas para anticipar accidentes que podrían dañar a los empleados si no perturban el lugar de trabajo o el entorno laboral. puede dañar (Polo, 2018). Se busca que la salud y seguridad ocupacional es estar trabajando para reducir los accidentes y la producción (Rodríguez, 2014). Por ello, la recuperación depende de la vida social y económica, lo que aumenta la productividad y contribuye al desarrollo social (DS N $\left.{ }^{\circ} \mathrm{OO} 5,2012\right)$. 


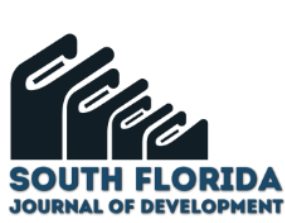

Por su parte, la variable riesgos laborales se cataloga como las posibles condicionantes dentro del centro laboral, esto puede derivar en accidentes laborales que pueden afectar o dañar la salud de los trabajadores a largo plazo (Benavides, Ruiz \& Gracia, 2007). Esto es intrínsecamente peligroso y aumenta la probabilidad de lesiones en el lugar de trabajo (Enríquez y Sánchez, 2012). No quiere decir que esto suceda, sólo predice situaciones peligrosas que condicionan de forma negativa la salud y seguridad de los colaboradores (Luna, 2012).

Por lo tanto, cualquier acto, circunstancia o causa que proporcione las circunstancias atenuantes necesarias para causar una lesión se considera una amenaza para la vida además de la dificultad causada por la lesión y resulta en una lesión en el trabajo. Salud u otras enfermedades. Los factores ocupacionales que son alterados por factores físicos, biológicos y ergonómicos (Yassi y Warshaw, 2003) dañan a los empleados debido a una o más de las siguientes tareas y peligros asociados: mayor oportunidad, limitaciones y sus consecuencias (Salvador, 2015). Por tanto, si los trabajadores resultan lesionados como consecuencia de sus actividades profesionales, es necesario determinar el alcance y la gravedad de su impacto (Agullo, 2015). Es un conjunto de accidentes y enfermedades que pueden ocurrir a raíz del trabajo o como efecto del trabajo (Creus, 2013).

El sistema debe tener en cuenta estos aspectos, especialmente facilitando una cultura de trabajo por medio de un proceso de mejora continua para hacer frente a situaciones que ponen en riesgo y ponen en peligro la seguridad y salud de los trabajadores. En resumen, la planificación, organización, implementación, evaluación y auditoría son todos pasos que permiten la gestión de riesgos de salud y seguridad ocupacional (Alli,2008).

Los factores asociados con diversas lesiones y enfermedades ocupacionales incluyen a) todas las condiciones físicas que causan una lesión ocupacional, como maquinaria, herramientas, equipos, pasillos y superficies, superficie donde se maniobran o transportan, equipos eléctricos, etc. Los vehículos provocan factores de seguridad, asfixia, cortes, colisiones, cortes, quemaduras, etc. b) Las causas derivadas de la naturaleza del trabajo, la manipulación del equipaje, el lugar de trabajo, el estrés mental que provoca irritación, el uso de la fuerza, que puede provocar insomnio y dolores de cabeza. c) Factores que se derivan de la organización del trabajo: Las actividades se consideran parte del trabajo y deben ser realizadas por los empleados en un momento determinado y durante un período de tiempo determinado, provocando estrés entre diferentes síntomas. d) Factores físicos de origen biológico, químico y físico: en general, las formas físicas están relacionadas con el ruido, la radiación, la luz, la humedad ambiental, etc. A diferencia de los elementos químicos, interfieren con el medio ambiente del trabajador, incluida la contaminación como polvo, aerosoles y humos, las cosas biológicas están formadas por virus, hongos y bacterias que causan enfermedades en los trabajadores (Cabaleiro, 2010). 


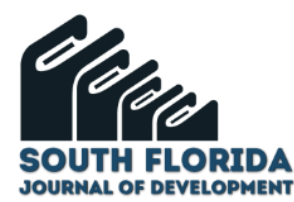

Por ejemplo, los riesgos laborales se pueden clasificar como: Peligros físicos que representan cambios repentinos de temperatura y ambiente, como ruido, humedad y ventilación. A menudo estamos expuestos a los peligros químicos de compuestos inorgánicas u orgánicas de procedencia natural o sintética que causan intoxicación, asfixia, irritación, etc. (Márquez, 2016).

Desde esta perspectiva, discutiremos algunos de los beneficios de la cobertura ocupacional. Algunos de los beneficios de la prevención de la seguridad laboral en este sentido son: a) reducir costos y pérdidas, b) mejorar el desempeño, la eficiencia y las capacidades de la organización, c) trabajar para la empresa d) difundir una cultura de confianza que respalde la motivación y el bienestar de los empleados, e) los clientes y proveedores, y mejorar la calidad de la empresa. imagen con respecto al trato recibido por el público. f) Mejorar la relación empleador-empleado. g) Incrementar la producción al reducir las enfermedades profesionales (Frick, 2000).

Para la primera dimensión física, identifique los factores medioambientales que requieren las propiedades físicas que puedan afectar al cuerpo humano, como el ruido, la luz, la temperatura, el fuego, la electricidad, la carga y la exposición a la radiación. Estos factores se consideran peligrosos y están asociados a una variedad de actividades que pueden conducir a cierto tipo de lesiones (Díaz, 2008). El segundo aspecto químico conocido de nuestro medio ambiente es la presencia de diferentes químicos en diferentes estados. Los sólidos, líquidos y gases pueden causar una variedad de daños en la piel y enfermedades crónicas o agudo cuando se expone sin precauciones (Fernando, 2012).

También hay un tercer aspecto biológico. Estos son hongos, bacterias, virus, etc. Lo que va en contra de la salud al provocar enfermedades ocupacionales, el cómo combatirlas es a través del tracto respiratorio, tracto digestivo, sangre, mucosidades e incluso alimentos, animales, etc. (MINSA, 2014). La ergonomía, por su parte, continúa siendo la cuarta dimensión. Se refiere al esfuerzo de los trabajadores por realizar actividades o cargas de trabajo que pueden causar fatiga y otro tipo de problemas de salud si se sienten abrumados. (García, 2018).

Finalmente, el quinto aspecto psicosocial afecta las partes emocionales, sociales y físicas asociadas a las labores que desempeña el trabajador o al entorno donde se desenvuelve, provocando estrés, problemas emocionales o físicos, existe la posibilidad de afectar adversamente la salud laboral y el desempeño normal (Gonzáles, 2003). En cuanto a la delimitación de indicadores dimensionales, podemos destacar lo siguiente:

a) Estructura y responsabilidad se refiere al reconocimiento de la estructura organizativa adoptada por los componentes de un sistema o sus propiedades específicas (Gilli, 2017).

b) La formación en este sector es de gran valor y es gravitante para las empresas ya que puede incrementar el nivel de producción y profesionalismo de todos los empleados (Rodríguez, 2010). Es en sí mismo un 


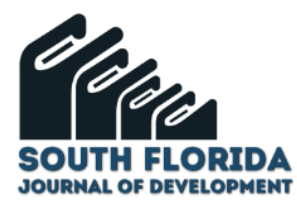

proceso de corto plazo que se desarrolla de manera continua y sistemática para adquirir habilidades, conocimientos y habilidades (Chiavenato, 2009).

c) Las medidas preventivas son varias medidas destinadas a proteger la seguridad y la salud de las personas ante diversas situaciones de amenaza, cuya función principal es mitigar los riesgos de las medidas preventivas adoptadas (Cortés, 2002).

d) La anticipación y temprana respuesta ante emergencias tiene prioridad sobre la idea de mejorar la seguridad y la salud de sus empleados mediante la implementación de procesos y actividades que trabajen con ello. Hay ciertos principios que se encuentran en un marco coherente y sistemático que eliminan los riesgos, para ello es necesario los procesos de prevención, protección de la salud y seguridad que reducen los riesgos para un trabajo altamente efectivo, asesoramiento, información, equidad, organizaciones justas y equilibradas (Moreno, 2010).

e) Requerimientos fijados se interrelacionan con los atenuantes o habilidades que tiene que poseer el usuario para dar solución a un problema o lograr un objetivo establecido. Así mismo se relaciona con la preparación o habilidad que debe poseer el operario para lo cual tiene que contar con plan sistémico o un componente para lograr cumplir con lo estipulado en el contrato, norma, etc. (Piattini, 1996). Esto implica mostrar una alternativa de solución de cara a una problemática (Sawyer y Kotonya, 2001).

f) La salud y seguridad ocupacional es una forma de proteger al trabajador, pero lo más importante es evitar situaciones peligrosas que pongan en peligro a sus empleados. En resumen, necesitamos mejorar el ambiente y las disposiciones en las que se desempeñan (Díaz, 2008).

g) Un accidente es cualquier caso de lesión física o psíquica que pueda sufrir un empleado en el desempeño de su trabajo (Montes, 1991). Así, la enfermedad ocupacional se refiere a cambios graves o crónicos en la salud como resultado de realizar un trabajo o exposición a sustancias físicas, químicas o biológicas. Los ejemplos incluyen problemas cardíacos y presión arterial alta.

h) La documentación requerida para esta actividad promueve la capacidad de evaluar los problemas de seguridad, abordar los riesgos asociados con las necesidades que afectan a las personas, los recursos y las misiones dentro de la organización y proponer soluciones alternativas. (Musgrave, 2011)

Es de tipo es básico o pura, obtiene la información a través de teorías para explicar el fenómeno de estudio y aumentando los conocimientos básicos de temas conceptuales (Valderrama, 2015). El diseño es una correlación descriptiva porque describe la relación existente entre dos o más variables y de un corte transversal porque se hace en un contexto particular (Pino, 2018). 


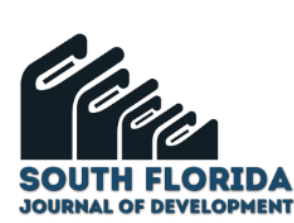

Se utilizó el método hipotético deductivo porque se propusieron hipótesis previas para dar una idea general del problema en consideración para explicar la importancia de los eventos desde un punto de vista cuantitativo y de uso estadístico. (Pino, 2018)

Dado que la población está conformada por 82 empleados, las muestras investigadas fueron tomadas de la empresa constructora Cobra Perú ubicada en San Isidro. Se utilizó el censo. Se usó la técnica de encuesta y herramienta de recopilación de datos es un cuestionario que contiene varias preguntas para recopilar datos de los encuestados y sirve como referencia. El cuestionario también sirve como referencia. Adaptado a los distintos controles de seguridad y salud laboral del cuestionario de (Neyra, 2018), (Alarcón y Maguiña,2018) sobre riesgos laborales. Para la realización del análisis de datos, se empleó la prueba de Kolmogorov - Srmirnov para la normalidad o coincidencias y, finalmente, empleó estadísticas descriptivas y de inferencia. Luego utiliza el software estadístico SPSS 22 para procesar la información y correlacionar la información obtenida por medio del estadístico no paramétrico Rho Spearman.

\section{RESULTADOS}

En relación a los resultados cuantitativos obtenidos son:

Tabla 1. Relación de las variables y sus dimensiones.

\begin{tabular}{|c|c|c|c|c|c|}
\hline Hipótesis & Variables*Correlación & $\begin{array}{c}\text { Rho- } \\
\text { Spearman } \\
\end{array}$ & $\begin{array}{c}\text { Significatividad- } \\
\text { Bilateral } \\
\end{array}$ & $\mathrm{N}$ & Nivel \\
\hline $\begin{array}{l}\text { Hipótesis } \\
\text { general }\end{array}$ & $\begin{array}{l}\text { Gestión de seguridad y } \\
\text { salud ocupacional * } \\
\text { riesgos laborales }\end{array}$ &, $472^{* * *}$ & 000 & 82 & Moderado \\
\hline $\begin{array}{l}\text { Hipótesis } \\
\text { especifica-1 }\end{array}$ & $\begin{array}{l}\text { Implementación y } \\
\text { operación * riesgos } \\
\text { laborales }\end{array}$ &, $418^{* *}$ &, 000 & 82 & Moderado \\
\hline $\begin{array}{l}\text { Hipótesis } \\
\text { especifica-2 }\end{array}$ & $\begin{array}{l}\text { Requisitos legales y } \\
\text { verificación* riesgos } \\
\text { laborales }\end{array}$ &, $435^{* *}$ &, 000 & 82 & Moderado \\
\hline $\begin{array}{l}\text { Hipótesis } \\
\text { especifica-3 }\end{array}$ & $\begin{array}{l}\text { Control e información de } \\
\text { documentos* riesgos } \\
\text { laborales }\end{array}$ &, $475^{* *}$ & 000 & 82 & Moderado \\
\hline
\end{tabular}

En función a los resultados obtenidos, se contrasta la hipótesis para contrastar la hipótesis nula frente a la hipótesis alternativa para ambas variables y sus dimensiones con el fin de establecer una relación entre las variables consideradas Rho de Spearman incluidas dentro de la hipótesis general. Gestión de la seguridad, salud y riesgo ocupacionales (Rho 0,472 y valor p 0,000) para obtener valores de correlación medianos. Hipótesis típica -1 (implementación y manipulación * riesgo ocupacional) que alcanza valores de correlación mediana (Rho 0,418 y valor p 0,000). Hipótesis típica 2 (requisitos legales 


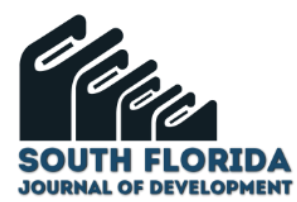

y validación * riesgo ocupacional) de puntuaciones medias (Rho 0,435 y valor p 0,000). La determinación del valor de correlación del riesgo ocupacional para la hipótesis específica -3 (información de auditoría y documento * medios (Rho 0.475 y valor p 0.000 )) dio un nivel de significancia de 0.01 para todos los resultados, con un nivel de significancia de $99 \%$.

\section{DISCUSIÓN}

En comparación con la hipótesis general, la gestión de la salud y la seguridad ocupacional se asoció con el riesgo ocupacional con una correlación moderada (Rho 0,472 y valor p 0,000). Roa (2017) afirmó que estos hallazgos requieren el establecimiento de sistemas de gestión de salud y seguridad a razón que las empresas no están preparadas y pueden verse eclipsadas por accidentes, Fabian (2017) mostró que se necesita una gestión de seguridad más eficiente para mejorar la labor de los empleados. Estas asociaciones difieren de las observadas por la correlación moderada en el diseño de Cobra Perú. Si bien se han producido algunos accidentes como consecuencia del sistema de gestión de salud y seguridad, también se han implementado algunas medidas de seguridad.

Según la hipótesis específica -1, la ejecución y el inicio de funcionamiento se asocian al riesgo laboral con valores de correlación mediana (Rho 0,418 y valor p 0,000). Estos resultados son inconsistentes con los de Poyehuanca (2019). Esto significa que debe crear un entorno saludable y saludable más adelante para crear un sistema que permita gestionar adecuadamente. Jiménez (2016) argumentó que la falta de educación es un sistema basado en un diagnóstico certero, por lo que es necesario introducir un sistema de gestión para mejorar la salud y seguridad de los empleados internos. También se habla de asociación. Para ello, estamos trabajando para establecer un sistema de gestión.

Según la hipótesis específica 2, Sobre los requerimientos legales y su aprobación tiene una correlación mediana (Rho 0,435 y valor p 0,000 ) con los requisitos legales se asocia con el riesgo laboral. Estos resultados son similares a los de Sarabia (2015), quien mencionó que el empleo de manuales sobre seguridad regula las medidas de protección del trabajador, pero Durán (2016) enfatiza las medidas preventivas para reducir las lesiones laborales. Por otro lado, Pinos (2015) tiene poca investigación sobre la ley de salud y seguridad ocupacional y está estrechamente relacionado con los derechos humanos, por lo que existen medidas de protección, pero todos estos términos están subordinados. Mientras tanto, no hay evidencia de su seguridad.

Finalmente, en particular, la hipótesis 3, gestión de documentos e información, se asocia al riesgo profesional con valores de correlación adecuados (Rho 0,475 y p-valor 0,000). Ante ello, Falcón (2019) sugiere que todos los riesgos laborales deben gestionarse por medio de la identificación y análisis de riesgos y enfermedades, por lo que los resultados pueden servir para demostrar la carencia de sistemas de 
gestión de salud y seguridad. De hecho, esto en Cobra Perú aumenta el nivel de riesgo laboral en la industria de la construcción.

\section{CONCLUSIONES}

Se ha comprobado lo siguiente que, entre administración de la seguridad y salud ocupacional y los peligros laborales, existe una relación moderada ( $\mathrm{Rho}=0.472$ ) con su p-valor $=0,000$.

Al analizar la relación que existe entre administración de la seguridad y salud ocupacional con sus dimensiones y la variable denominada riesgos laborales se logró demostrar que hay una relación directa e importante al conseguir valores de 0.418 , de 0.435 y de 0.00 por intermedio del estadístico Rho de Spearman correspondientes respectivamente a las dimensiones: puesta en marcha y operación, dimensión llamada condiciones legales y control e información de los documentos con correlaciones moderadas.

Dichas variables sometidas a la investigación deben formar parte del trabajo y estar dentro de los parámetros de investigación que deberían ser promovidas por todas las instituciones que colaboran con el estudio.

Realizar revisiones a varias investigaciones con similares problemáticas investigadas por otros grupos de interés y abarcar otras localidades a nivel nacional e internacional. 


\section{REFERENCIAS}

Alarcón, S., y Maguiña, K. (2018) Implementación de un sistema de gestión de seguridad y salud ocupacional para disminuir los riesgos laborales en el Centro Médico Villa (Tesis de maestría) Universidad César Vallejo.

Agullo, J. (2015). Prevención de riesgos laborales. Madrid, España: Ediciones parainfo

Apaza, R. (2012) Seguridad y salud ocupacional. España, Barcelona: Innova

Arévalo, J. y Molano, N. (2013) Innovar. Volumen 23, N48, ISSN 2248-6968

Benavides, F.; Ruiz, C y García, A. (2007) Salud laboral.2 da edición. Barcelona:Masson. España. P.168.

Cabaleiro, V. (2010). Prevención de riesgos laborales. ( $3^{\circ}$ ed.). Recuperado de https://books.google.com/books?id=akZ-I4YMMZ8C

Chiavenato I. (2009) Gestión del Talento Humano 3ra edición, Editorial Mc Graw-Hi 11.

Cortés J., (2002) Seguridad e higiene del trabajo técnicas de prevención de riesgos laborales,3ra Edición, editorial Tébar, México.

https://www.oficemen.com/wp-content/uploads/2017/05/Guia-PRL-capitulos-4-y-5.pdf

Creus, A. (2013). Técnicas para la prevención de riesgos laborales. Barcelona, España: Marcombo.

Díaz, P. (2008). Prevención de riesgos laborales. $2^{\circ}$ ed. España: Paraninfo. 117 pp.

Díaz, T.(2008) Manual para la formación en prevención de riesgos laborales $5^{\circ}$ ed. España: Lex Nova, $426 \mathrm{pp}$.

Durán (2016) Implementación de medidas de prevención y control de los riesgos ergonómicos del personal de enfermería del servicio de neurocirugía del Hospital Carlos Andrade Marín de Quito. (Tesis de maestría) Escuela Politécnica Nacional de Quito.

Enríquez, A. y Sánchez, J. (2012) Sistema de Gestión de la Seguridad y Salud en el trabajo. $3^{\text {a }}$. Ed. Madrid: Fundación Confemetal, 313 p.

Fabián, E. (2017) Sistema de gestión en seguridad y salud ocupacional en la planta de Yauris (Tesis de maestría) Universidad Nacional del Centro del Perú.

Falcón,I. (2019) Diseño de un sistema de gestión en seguridad y salud ocupacional para el control de riesgos laborales en housekeeping - hotel Casa Blanca, Chanchamayo.(Tesis de maestría)Universidad San Martín de Porres.

Fernando, L. (2012) Prevención de riesgos laborales. España: Fundación Vértice emprende, 414 pp

Frick, K. (2000). Systematic. Oxford: Pergamon

García, E. (2018) Seguridad y Salud. España: Nobel, 163 pp. 
Gilli, J. (2017). Claves de la estructura organizativa. Buenos aires: Granica

González, R. (2003) Manuel básico prevención de riesgos laborales. España: Paraninfo, 224 pp

ISEM (2011) Seguridad Minera. SG - SST; Una herramienta para la mejora continua, Semana 16 p 4

Jiménez,N. (2016) Implantación de un sistema de gestión de seguridad y salud ocupacional en comercial molinera San Luis, Lambayeque. (Tesis de maestría) Universidad Nacional de Trujillo.

Luna, F. (2012) Prevención de riesgos laborales. Editorial Vértice, España.

Márquez, M.(2016) "Factores de riesgo relevantes vinculados a molestias musculoesqueléticas en trabajadores industriales", Salud de los Trabajadores vol.24 no.2 Maracay.

Mearns, K.; Sean,M. y Rhona,F.(2003) "Safety climate, safety management practice and safety performance in offshore environments", Safety Science, Elsevier, vol. 41, pp. 641-680

Merino, J. (2016). Teoría para la prevención y análisis de accidentes basada entrayectorias dinámicas. Caracas: Bolivariana.

Ministerio de Salud (2014). Guía sobre prevención de riesgos psicosociales. Recuperado de http://www.digesa.minsa.gob.pe/noticias/Julio2014/nota119.asp

Montes, E. (1991) Tratado de seguridad e higiene. Universidad Pontificia de Comillas.

Moreno, M. (2010) Manual para la formación en prevención de los riesgos laborales. Editorial: Lex Nova Musgrave, G., Ph.D, A., y Tommaso S,(2011) Safety Design for Space Systems

Neyra, G. (2018) Nivel de Conocimiento del Sistema de Gestión de Seguridad y Salud en el Trabajo del Centro Materno Infantil Santa Luzmila II (Tesis de maestría) Universidad César Vallejo.

Nkrumah, E.; Gyabeng, E. y Akoto, L. (2020) The state of occupational health and safety management frameworks (OHSMf) and occupational injuries and accidents in the ghanaian oil and gas industry: assessing the mediating role of safety knowledge. En BioMed Research International · March 2020 DOI: $10.1155 / 2020 / 6354895$

Pino, R. (2018) Metodología de la investigación. 5ta Ed. Editorial San Marcos, Lima-Perú.

Piattini,G. (1996). Análisis y diseño detallado de aplicaciones informáticas de gestión. Rama. Editorial Ciclos informativos.

Pinos, L. (2015) Gestión de riesgos laborales en las prácticas de responsabilidad social corporativa en el Ecuador (Tesis de doctorado) Universidad de Huelva. España.

Poyehuanca, M. (2019) Desarrollo e implementación de un sistema de gestión de seguridad y salud ocupacional en el trabajo para la empresa prestadora de saneamiento Nor Puno S.A. (Tesis de maestría) Universidad Nacional del Altiplano, Puno, Perú. 
Polo, S. (2018). Sistema de gestión en seguridad y salud en el trabajo. Comité Paritario de Seguridad y Salud en el Trabajo.

Roa, D. (2017) Sistemas de Gestión en Seguridad y Salud en el Trabajo (SG-SST) (Tesis de maestría) Universidad Nacional de Colombia.

Robson, L. (2010). Something might be missing from occupational health and safety. Journal, 51

Rodríguez, F. (2014) La seguridad y salud ocupacional como elemento de la gestión del potencial humano. México. Editorial Planeta.

Rodríguez, M. (2010). Administración de la capacitación. McGraw-Hill Interamericana.

Salvador, J. (2015). Prevención de riesgos laborales. Lima: Fundación Confemetal

Sarabia,C. (2015) Gestión de riesgos laborales en la fábrica de dovelas del Proyecto Hidroeléctrico Coca Codo Sinclair: Manual de seguridad. Universidad Nacional del Chimborazo, Ecuador.

Sawyer, P.y Kotonya, G. (2001) Software Requirements. Edition 2 Guide to the Software Engineering Body of Knowledge SWEBOK. IEEE-CS Press.

Yassi, A. y Warshaw, L. J. (2003). Asistencia sanitaria: Naturaleza y problemas de salud en el trabajo. Asistencia sanitaria, 97(2); 2 - 10.

Valderrama, S. (2015). Pasos para elaborar proyectos de investigación científica. Lima: Ed. San Marcos. 